Article

\title{
A Co-Precursor Approach Coupled with a Supercritical Modification Method for Constructing Highly Transparent and Superhydrophobic Polymethylsilsesquioxane Aerogels
}

\author{
Chaoshuai Lei ${ }^{1,2}{ }^{(\mathbb{D})}$, Junning $\mathrm{Li}^{2}$, Chencheng Sun ${ }^{2}$, Hailong Yang ${ }^{2}$, Tao Xia ${ }^{1,2}$, Zijun Hu ${ }^{1,2, *}$ \\ and Yue Zhang ${ }^{1, *}$ \\ 1 School of Materials Science and Engineering, Beihang University, Xueyuan Road 37, Beijing 100191, China; \\ leichaoshuai@126.com (C.L.); xiatao@buaa.edu.cn (T.X.) \\ 2 National Key Laboratory of Advanced Functional Composite Materials, Aerospace Research Institute of \\ Materials and Processing Technology, Beijing 100076, China; ljn1212@163.com (J.L.); \\ sunyahua2005@163.com (C.S.); yhl20032003@126.com (H.Y.) \\ * $\quad$ Correspondence: 13522240902@163.com (Z.H.); zhangy@buaa.edu.cn (Y.Z.); Tel.: +86-183-1076-1101 (Z.H.)
}

Received: 28 February 2018; Accepted: 21 March 2018; Published: 30 March 2018

\begin{abstract}
Polymethylsilsesquioxane (PMSQ) aerogels obtained from methyltrimethoxysilane (MTMS) are well-known high-performance porous materials. Highly transparent and hydrophobic PMSQ aerogel would play an important role in transparent vacuum insulation panels. Herein, the co-precursor approach and supercritical modification method were developed to prepare the PMSQ aerogels with high transparency and superhydrophobicity. Firstly, benefiting from the introduction of tetramethoxysilane (TMOS) in the precursor, the pore structure became more uniform and the particle size was decreased. As the TMOS content increased, the light transmittance increased gradually from $54.0 \%$ to $81.2 \%$, whereas the contact angle of water droplet decreased from $141^{\circ}$ to $99.9^{\circ}$, ascribed to the increase of hydroxyl groups on the skeleton surface. Hence, the supercritical modification method utilizing hexamethyldisilazane was also introduced to enhance the hydrophobic methyl groups on the aerogel's surface. As a result, the obtained aerogels revealed superhydrophobicity with a contact angle of $155^{\circ}$. Meanwhile, the developed surface modification method did not lead to any significant changes in the pore structure resulting in the superhydrophobic aerogel with a high transparency of $77.2 \%$. The proposed co-precursor approach and supercritical modification method provide a new horizon in the fabrication of highly transparent and superhydrophobic PMSQ aerogels.
\end{abstract}

Keywords: polymethylsilsesquioxane aerogels; co-precursor; supercritical modification; transparency; superhydrophobicity

\section{Introduction}

Silica aerogels have gained extensive attention in the past decades owing to their extraordinary properties, such as their low density, nanoporous structure, high specific surface area, high optical transparency, and extremely low thermal conductivity in some chemical systems [1]. For these unique characteristics, silica aerogels are presently of interest in thermal insulation [2], acoustic insulation [3], energy-saving windows [4], catalysts [5], and drug delivery systems [6]. Generally, silica gels are synthesized from tetra-alkoxysilane precursors through the sol-gel route and the monolithic aerogels are obtained after draining the pore liquid with supercritical drying [7-9]. However, because of the brittle tetra-functional siloxane network, most of the previously reported silica aerogels are usually fragile, hydrophilic, or opaque. 
Scientists have made great efforts to improve the properties of silica aerogels. One of the promising approaches was utilizing alkyltrialkoxysilane instead of traditional tetra-functional silicon alkoxides such tetramethylorthosilicate (TMOS) or tetraethylorthosilicate (TEOS) [10,11]. As one kind of alkyltrialkoxysilane, MTMS was usually utilized as the precursor while the obtained flexible and hydrophobic aerogels were opaque due to the phase separation [12,13]. Kanamori et al. reported the first successful fabrication of transparent monolithic PMSQ aerogels by exploiting appropriate surfactants to prevent the macroscopic phase separation [14]. However, the substituent methyl groups in MTMS have steric effects that provide barriers for hydrolysis and condensation reactions of $\mathrm{Si}-\mathrm{OCH}_{3}$ groups in the sol-gel process [15]. Further hydrolysis and condensation of unhydrolyzed groups during the temperature supercritical drying process will lead to the pore structure coarsening with larger particle and pore size. This will result in the low transparency according to the Rayleigh scattering mode $[16,17]$. One of the prevalent approaches to improve the transparency of the aerogels was introducing TMOS together with MTMS in a sol-gel route, namely the co-precursor method $[18,19]$. Benefiting from the low energy barriers, hydrolysis and condensation reactions of TMOS are complete $[20,21]$. Then the particles in aerogels remain small and uniform after high temperature supercritical drying process, which is beneficial to the enhancement of the transparency of aerogels.

The superhydrophobicity of the aerogels were usually achieved using the surface modification of the skeleton with specific chemical groups such as hexamethyldisilazane (HMDZ). The modification procedure can be performed during the aging process or supercritical drying process or carried out as a post-treatment by gas phase reactions with the formed aerogel network [22-24]. The lengthy and tedious aging modification usually took several days or weeks due to the solvent-exchange process [25]. Gas phase modification of the as-prepared aerogels had a negative effect on the pore structure due to the gas condensation. In contrast, supercritical modification technology during the drying process not only rendered modification much faster and more complete but also caused very slightly differences in the pore structure [26,27]. Incorporating hydrophobic groups with the hydroxyl on the silica surface, the supercritical modification would endow the monolithic aerogels with hydrophobicity [23].

In this work, highly transparent PMSQ aerogels were prepared by the co-precursor method. With the increase of TMOS content in the precursor, the particle size and pore diameter of the PMSQ aerogel were decreased gradually due to the relatively complete hydrolysis and condensation reactions. The light transmittance of the obtained aerogels was up to $80 \%$. Meanwhile, superhydrophobicity of the aerogel was realized by modifying the surface hydroxyl groups with hexamethyldisilazane through supercritical drying process. The modified aerogels with a contact angle of $155^{\circ}$ retained almost the same pore structure compared with the aerogels without modification. Therefore, the PMSQ aerogel possesses a high transparency of $77.2 \%$. The highly transparent, superhydrophobic aerogels would extend their practical application such as transparent insulation window.

\section{Materials and Methods}

\subsection{Materials}

Methyltrimethoxysilane (MTMS), tetramethoxysilane (TMOS) and hexamethyldisilazane (HMDZ) were obtained from Adamas Reagent Co., Ltd. (Shanghai, China). Acetic acid, ethanol, and ammonia $\left(\mathrm{NH}_{3} \cdot \mathrm{H}_{2} \mathrm{O}\right)$ were purchased from Beijing Chemical Works (Beijing, China). Surfactant n-hexadecyltrimethylammonium chloride (CTAC) was supplied by Sinopharm Chemical Reagent Co., Ltd. (Shanghai, China). All the chemical reagents were analytical reagents and used as received without further purification. 


\subsection{Preparation of Silica Aerogels}

Silica hydrogels were synthesized with MTMS and TMOS as co-precursors through a two-step, acid-base catalyzed sol-gel process. In a typical synthesis, $60 \mathrm{~mL}$ of diluted aqueous acetic acid $(5 \mathrm{mM}), 0.6 \mathrm{~g}$ of surfactant CTAC were mixed in a glass beaker. MTMS and TMOS were subsequently added with vigorous stirring $30 \mathrm{~min}$ for hydrolysis reaction. After that, $\mathrm{NH}_{3} \cdot \mathrm{H}_{2} \mathrm{O}$ dissolved in $30 \mathrm{~mL}$ deionized water was poured into the mixed solution under stirring. The molar ratio of the starting materials was (MTMS + TMOS): $\mathrm{H}_{2} \mathrm{O}$ :acetic acid:CTAC: $\mathrm{NH}_{3} \cdot \mathrm{H}_{2} \mathrm{O}=1: 24: 1.4 \times 10^{-3}: 8.9 \times 10^{-3}$ : $3.8 \times 10^{-3}$. The temperature of hydrolysis and condensation steps was $30^{\circ} \mathrm{C}$. The obtained hydrogels were then aged for 3 days to complete the condensation at $60^{\circ} \mathrm{C}$, followed by washing with ethanol three times ( $8 \mathrm{~h}$ each time) to remove the residual surfactant and chemicals. The washed wet gels were dried by ethanol supercritical method at $255^{\circ} \mathrm{C}$ and $8 \mathrm{MPa}$. The proportion of TMOS (x) in precursor was varied from $0 \%$ to $50 \%$ in increments of $10 \%$. The obtained aerogels were labeled as T0, T10, T20, T30, T40, and T50, respectively. For the sample T30, the supercritical fluid modification was implemented using HMDZ/ethanol solution with volume ratio of 1:50. The sample was marked as T30-H.

\subsection{Characterization of Materials}

The bulk density of the aerogels was measured from the weight/volume ratio of the monoliths. The microstructure of the aerogels was studied using field-emission scanning electron microscopy (FE-SEM, S-4800, Tokyo, Japan).

Specific surface area and pore size distribution (PSD) were obtained from $\mathrm{N}_{2}$ adsorption-desorption (Autosorb-iQ, Boynton Beach, FL, USA). The samples were outgassed at $150{ }^{\circ} \mathrm{C}$ prior to the adsorption-desorption measurement. The specific surface areas of the aerogels were calculated by the Brunauer-Emmett-Teller (BET) method, based on the amount of $\mathrm{N}_{2}$ adsorbed at pressure $0.05<\mathrm{P} / \mathrm{P}_{0}<0.3$. The pore size distributions of the samples were determined by desorption branch of the isotherm by employing the BJH method.

For light transmittance measurements, a UV-vis spectrometer UV-3600 (Shimadzu Corp., Kyoto, Japan) was employed. The obtained transmittance data at $550 \mathrm{~nm}$ was normalized into those corresponding to the thickness of $10 \mathrm{~mm}$ using the Lambert-Beer equation.

The surface wettability of PMSQ aerogels was evaluated by static contact angle measurement, using a Contact Angle System OCA (Dataphysics, Stuttgart, Germany). A droplet (1 $\mu \mathrm{L})$ of deionized water was placed very slowly on the surface of the hydrophobic aerogel.

The chemical composition of silica aerogels was characterized with Fourier transform-infrared spectroscopy with the attenuated reflection model (FTIR-ATR, Perkin-Elmer, Waltham, MA, USA).

Compression tests were performed on a monolithic cylindrical sample using a Universal Testing System (CTM5105, Guangzhou, China). The sample was placed between the testing plates and was compressed at a speed of $1 \mathrm{~mm} / \mathrm{min}$ to $50 \%$ strain and this position held for $10 \mathrm{~s}$ before the piston was retracted. Three replicates were performed for different samples in one group. The resilience of the aerogels is defined as the ratio of the final dimension after release of compressive stress to the original scale.

\section{Results and Discussion}

\subsection{Structural Characterization of the PMSQ Aerogels Prepared by the Co-Precursor}

Figure 1 shows the influence of TMOS content on the radial shrinkage and density of the PMSQ aerogels. With the increase of TMOS content in the precursor from $0 \%$ to $50 \%$, the radial shrinkage of the aerogels decreases from $7.45 \%$ to $3.56 \%$. Correspondingly, the density of the aerogel decreases from $0.150 \mathrm{~g} / \mathrm{cm}^{3}$ to $0.120 \mathrm{~g} / \mathrm{cm}^{3}$. Shrinkage of the aerogels during the high temperature supercritical drying process was resulted from the rearrangement reactions in the gel network, which is ascribed to the further hydrolysis and condensation of unhydrolyzed $\mathrm{Si}-\mathrm{OCH}_{3}$ groups. For the aerogel T0 
obtained from MTMS as the sole precursor, the steric effect of the methyl group provided barriers for hydrolysis and condensation reactions [15]. Continued reactions during high temperature drying process caused the PMSQ aerogel to have high radial shrinkage of $7.45 \%$. The reaction of TMOS is more complete in the sol-gel process because of the low energy barriers in hydrolysis and condensation reactions [28]. As a result, the radial shrinkage of the aerogel T50 decreased to $3.56 \%$ when the TMOS content was $50 \%$ in the precursor.

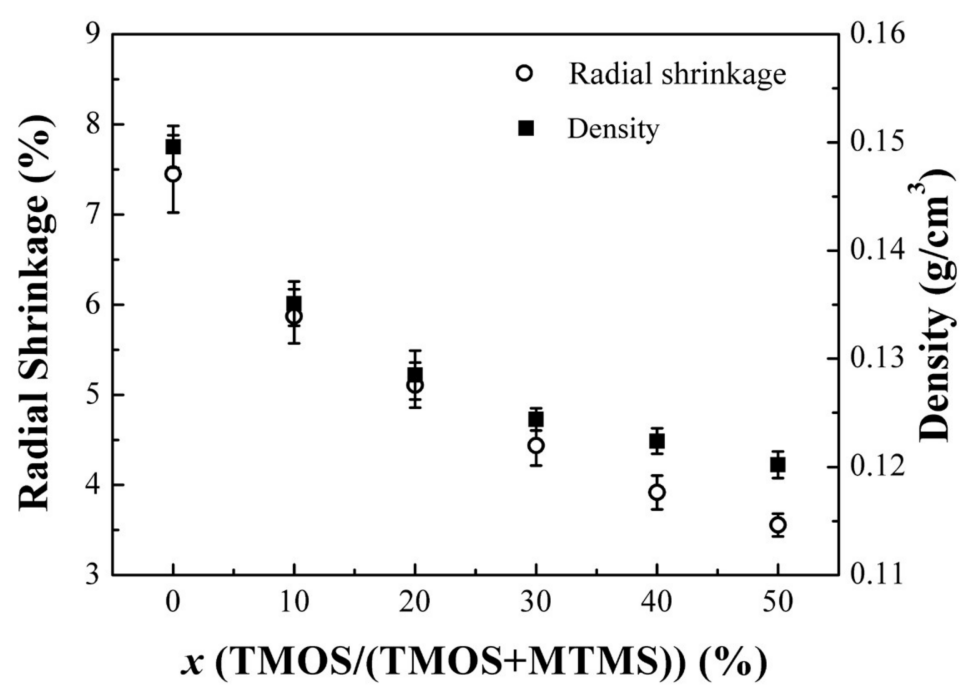

Figure 1. The radial shrinkage and density of aerogels with varied amount of TMOS.

Figure 2 presents the SEM images of the aerogels prepared with different contents of TMOS. All the aerogels exhibit 3D continuous network structure which is comprised of nano-scale particles and pores. It is found that the particle size and pore diameter in the aerogels prepared via the co-precursor method are smaller than those in the aerogel T0. Meanwhile, more uniform pore structure was obtained via the co-precursor method. Pore structures were further confirmed in Figure 3. $\mathrm{N}_{2}$ adsorption-desorption isotherms in Figure 3a reveal type IV nature and H1 hysteresis loops according to the IUPAC classification, demonstrating that all the aerogels are typical mesoporous materials distributions [29]. The average pore size distributions in Figure $3 \mathrm{~b}$ reveal that the pore diameter was decreased from $13 \mathrm{~nm}$ of $\mathrm{T} 0$ to $8.7 \mathrm{~nm}$ of T50. A gradual increase in the specific surface areas with increasing TMOS content is depicted in Figure 3c. Aerogel T50 has the highest specific surface area of $688.6 \mathrm{~m}^{2} / \mathrm{g}$, which is primarily attributed to the smaller particles in the aerogel [30]. While the specific surface area of aerogel T0 is only $556.5 \mathrm{~m}^{2} / \mathrm{g}$. As we know, less-polar MTMS oligomers are incompatible with any low-molecular weight solvents [14]. Then, surfactants such as CTAC always serve as a structure directing agent to realize mesoporous structure in the aerogels through inhibiting phase separation [31]. The introduced TMOS is soluble in the solvent water due to the rapid hydrolysis reaction [21]. The homogeneous solution in the sol-gel process contributes to the formation of small particles and uniform pore structure. The differences in chemical composition and pore structure will result in differences in the optical transparency, hydrophobicity, and mechanical properties of the aerogels. 


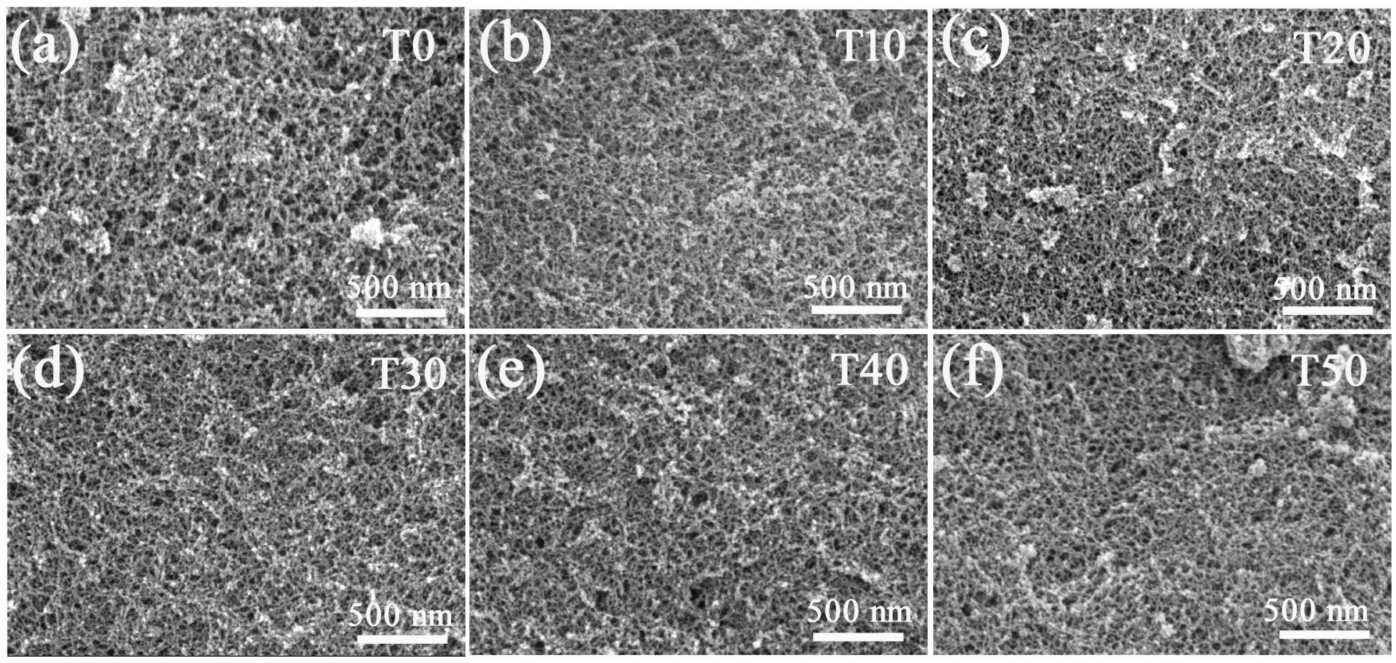

Figure 2. SEM images of the aerogel samples; (a) T0, (b) T10, (c) T20, (d) T30, (e) T40, and (f) T50.
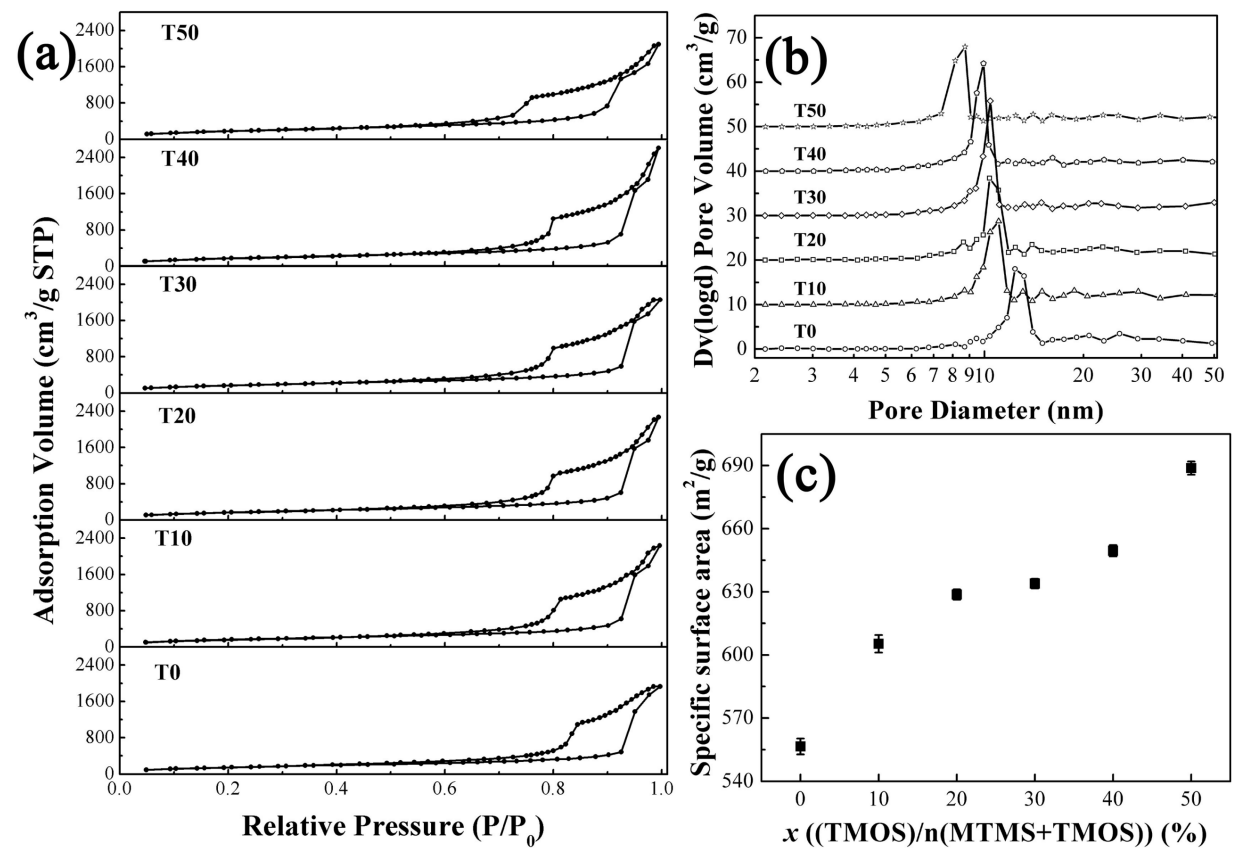

Figure 3. $\mathrm{N}_{2}$ adsorption-desorption isotherms (a); the pore size distributions (b) and the specific surface area of the aerogels $(\mathbf{c})$.

\subsection{The Properties of the PMSQ Aerogels Prepared by the Co-Precursor}

\subsubsection{The Transparency of Aerogels with Varied TMOS Content}

The photographs of the aerogel monoliths are shown in Figure 4a. Obviously, the transparency of the aerogels is improved significantly after the addition of TMOS. Light transmittance in Figure $4 \mathrm{~b}$ increases from $54.0 \%$ of $\mathrm{T} 0$ to $81.2 \%$ of $\mathrm{T} 50$, which is comparable to the value of traditionally derived silica aerogels [32]. In fact, the light transmittance of aerogel T30 is already up to $78.1 \%$, where the molar ratio of TMOS is $30 \%$. The dominant light scattering mode is Rayleigh scattering in the mesoporous aerogels and the particles and pores act as scattering centers [19]. Smaller particle size and pore diameter can decrease the scattering of light, leading to the transmittance increase as predicted by 
the scattering mode. The co-precursor method with introduction of TMOS plays a significant role in reducing particle size and improving the transparency of PMSQ aerogels.
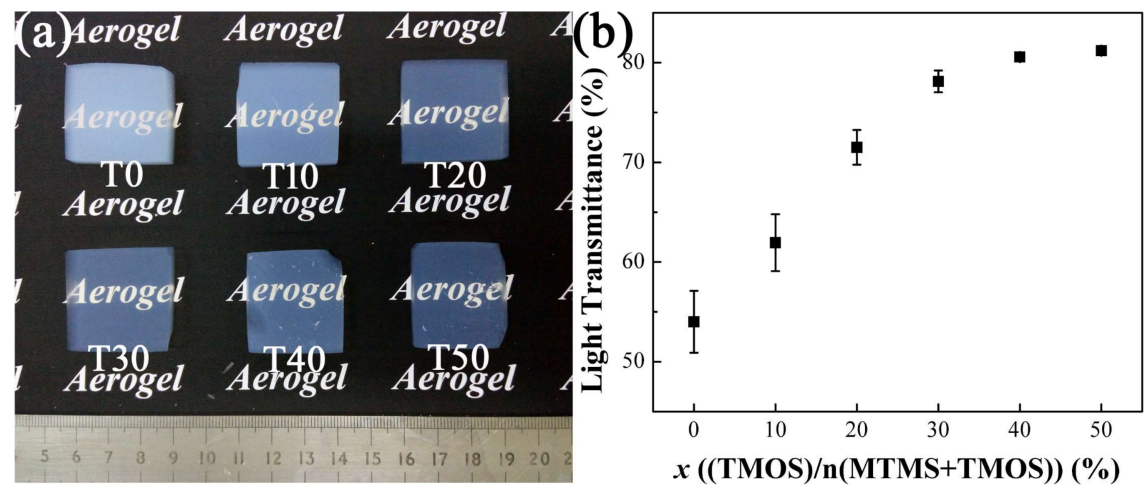

Figure 4. Photographs of the monolithic aerogels (a) and the light transmittance at $550 \mathrm{~nm}$ wavelength (b).

\subsubsection{The Hydrophobicity of Aerogels with Varied TMOS Content}

The hydrophobicity of aerogels is very importance for their wide used in practical applications [33]. However, the hydrophobicity of the aerogels will decrease with increasing TMOS due to the increase of hydroxyl group on the particle surface. The contact angle of the water droplet in Figure 5a decreased from $141^{\circ}$ to $99.9^{\circ}$ when the molar ratio of TMOS in precursor increased from $0 \%$ to $50 \%$. Chemical structures in the aerogels have been confirmed by FTIR-ATR spectroscopy as shown in Figure 5b. The peaks at $2980 \mathrm{~cm}^{-1}$ and $1270 \mathrm{~cm}^{-1}$ represent the stretching of the $\mathrm{Si}-\mathrm{CH}_{3}$ [34]. The stretching vibration peak of $\mathrm{Si}-\mathrm{C}$ can be observed at $840 \mathrm{~cm}^{-1}$ [35]. Notably, the characteristic peaks of $\mathrm{Si}-\mathrm{CH}_{3}$ gradually become weaker with the increase in TMOS content. Results state that the methyl group in the aerogel skeleton is gradually replaced by the hydroxyl group with the introduction of TMOS, which results in decreased hydrophobicity. Surface modification should be carried out to improve the hydrophobicity of PMSQ aerogels.
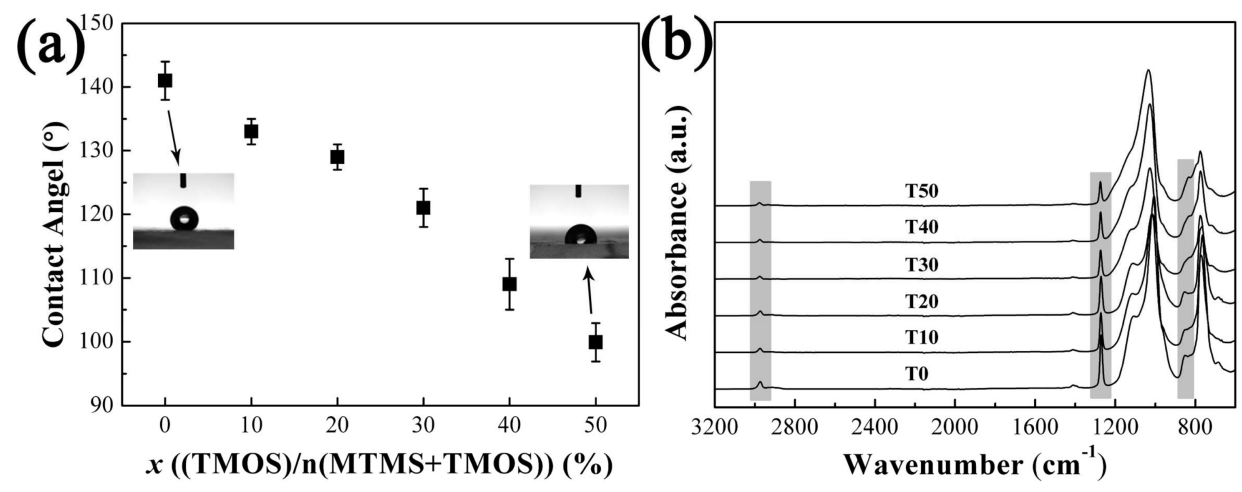

Figure 5. The contact angle of water droplet (a) and FTIR-ATR spectra of all the samples (b).

\subsubsection{The Mechanical Properties of Aerogels with Varied TMOS Content}

The mechanical properties of the aerogels were evaluated by uniaxial compression tests. The stress-strain curves in Figure 6a indicate that these PMSQ aerogels could endure $~ 50 \%$ strain without any damage, illustrating that the skeleton remains flexible in the aerogels obtained from the co-precursor method. The compressive strength is enhanced gradually with increasing the molar ratio of TMOS to $30 \%$. Then aerogel T30 possesses the maximum compressive strength of $0.76 \mathrm{MPa}$ at $50 \%$ strain. However, further increase in the proportion of TMOS in precursor lead to a significant 
reduction in the compression strength. The compressive strength of aerogel T50 decreases to $0.4 \mathrm{MPa}$ which is lower than the value of aerogel T0. Meanwhile, the resilience of the aerogels increased with increasing TMOS proportion to reach a maximum value of $76 \%$ at sample T30, from which it decreased to $60 \%$ of T50, as shown in Figure $6 \mathrm{~b}$. The improvement of mechanical properties is derived from the increase of silanol groups that are closely located to each other in the skeleton [14]. The tetra-functional siloxane network would improve the rigidity of the skeleton. However, excess introduction of TMOS can reduce the methyl groups significantly and make the skeleton become brittle. When stress is applied to the aerogel skeleton, the irreversible deformation would damage the framework. Furthermore, the repulsive interactions between hydrophobic moieties will be weakened with the decrease of methyl groups. Therefore, the mechanical properties of aerogel T30 are superior to those of the other aerogels.
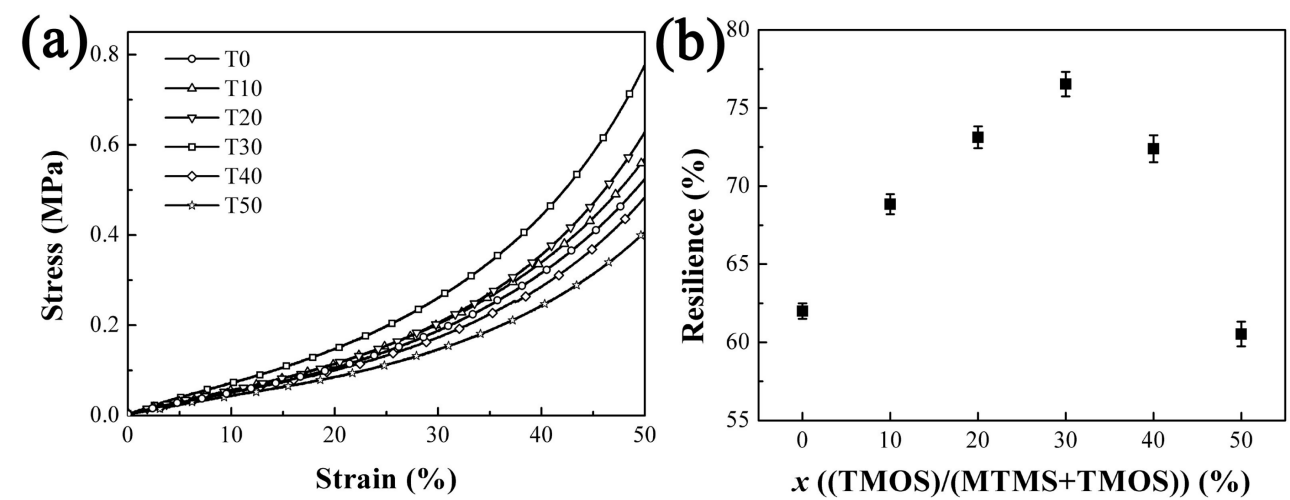

Figure 6. Compressive stress versus strain curves of the PMSQ aerogels (a) and the resilience of the samples compressed to $50 \%$ strain (b).

\subsection{The Supercritical Modification of the PMSQ Aerogel}

In order to obtain high-transparency, superhydrophobic, and elastic PMSQ aerogels, the wet gels of T30 were chosen for supercritical fluid modification with hexamethyldisilazane. The modifier HMDZ would react with the $-\mathrm{OH}$ on the skeleton surface, which results in methyl coatings on the nanoparticles. Therefore, the condensation of the neighboring $-\mathrm{OH}$ on the silica surface during drying is effectively restricted. Then, shrinkage of silica wet gels during drying are effectively inhibited. After saturation with methyl groups, the aerogel surface cannot be covalently attached by a chemical bond [23]. Structural comparison of aerogel T30 and modified aerogel T30-H is shown in Figure 7. It is worth noting that these two samples hold the same uniform pore structure with small particles and pore size, as shown in Figure 7a. The $\mathrm{N}_{2}$ adsorption-desorption isotherms and pore size distributions display that pore structures of T30-H are affected very slightly by the methyl groups loading on the aerogel skeleton surface.

There is no significant difference in microstructure between the modified aerogel T30- $\mathrm{H}$ and the unmodified aerogel T30. Aerogel T30-H retained a high transparency of $77.2 \%$, which is comparable to the value of aerogel T30 as shown in Figure 8a. The contact angle of T30-H was determined to be $155^{\circ}$, which is indicative of superhydrophobicity. The chemical structure analysis in Figure $8 \mathrm{~b}$ further supports the covalent bonding of the modifier with the aerogel surface. FTIR-ATR spectroscopy shows that the characteristic peaks of Si-CH $\left(2980 \mathrm{~cm}^{-1}, 1270 \mathrm{~cm}^{-1}\right.$ and $\left.840 \mathrm{~cm}^{-1}\right)$ obviously become stronger with the modification process, which signifies the reaction between $\mathrm{HMDZ}$ and surface $-\mathrm{OH}$ groups. Besides, the $-\mathrm{CH}_{3}$ coating endows aerogel $\mathrm{T} 30-\mathrm{H}$ with higher mechanical properties, compared with the aerogel without modification. The compressive strength of aerogel T30-H is $0.90 \mathrm{MPa}$ and the resilience of the aerogel is $85 \%$. This improvement in the mechanical properties is attributed to the repulsive interactions between the surface $-\mathrm{CH}_{3}$ groups, which promote the spring-back effect. These 
characteristics of high transparency, superhydrophobicity, and elasticity are important for the practical application of these materials, such as in transparent vacuum insulation panels.
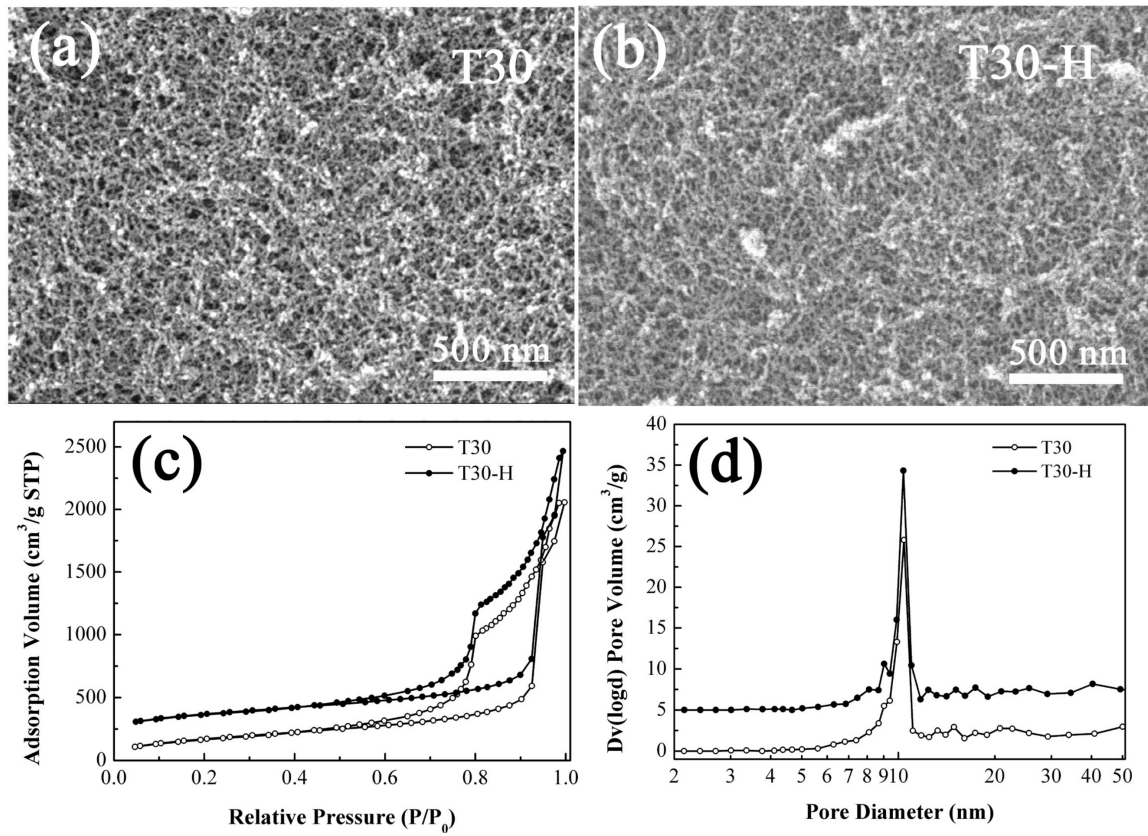

Figure 7. The SEM images of the aerogels T30 and T30-H (a,b); $\mathrm{N}_{2}$ adsorption-desorption isotherms (c) and the pore size distributions (d) of the aerogels T30 and T30-H.
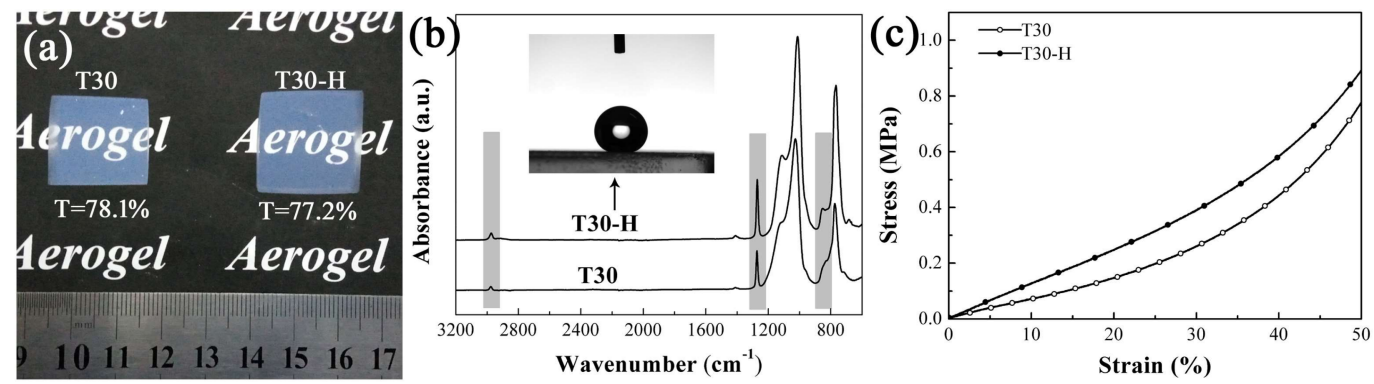

Figure 8. Photographs of the monolithic aerogels T30 and T30-H (a); FTIR-ATR spectra (b) and compressive stress versus strain curves of aerogels $\mathrm{T} 30$ and $\mathrm{T} 30-\mathrm{H}$ (c).

\section{Conclusions}

Highly transparent, superhydrophobic, and elastic PMSQ aerogels have been prepared via the co-precursor and supercritical modification method. The introduction of TMOS plays an important role in the structural stability of the aerogel during the high temperature supercritical drying process. With the increase in TMOS content, the particle and pore size of the PMSQ aerogel gradually become smaller. The light transmittance increases to $78.1 \%$ when the molar ratio of TMOS is $30 \%$. The superhydrophobicity of the aerogel is achieved by reacting the surface hydroxyl groups with HMDS through supercritical fluid modification. Modified aerogels revealed a high contact angle of $155^{\circ}$. The mechanical properties of the aerogels were slightly improved due to the repulsive interactions between the methyl groups. More importantly, the pore structures of PMSQ aerogels with and without modification stay the same. Therefore, the modified aerogel retains a high transparency of $77.2 \%$. These extraordinary characteristics will extend the range of applications for PMSQ aerogels. 
Acknowledgments: The authors thank financial supports from the National Natural Science Foundation of China (51672014, 51576058).

Author Contributions: C.L., Z.H. and Y.Z. conceived and designed the experiments; C.L. and T.X. performed the experiments; H.Y. analyzed the data; C.S. contributed reagents/materials/analysis tools; C.L. and J.L. wrote the paper.

Conflicts of Interest: The authors declare no conflict of interest.

\section{References}

1. Soleimani Dorcheh, A.; Abbasi, M.H. Silica aerogel; synthesis, properties and characterization. J. Mater. Process. Technol. 2008, 199, 10-26. [CrossRef]

2. Li, Z.; Cheng, X.D.; He, S.; Shi, X.J.; Yang, H.; Zhang, H.P. Tailoring thermal properties of ambient pressure dried MTMS/TEOS co-precursor aerogels. Mater. Lett. 2016, 171, 91-94. [CrossRef]

3. Feng, J.D.; Le, D.; Nguyen, S.T.; Nien, V.T.C.; Jewell, D.; Duong, H.M. Silica-cellulose hybrid aerogels for thermal and acoustic insulation applications. Colloids Surf. A Physicochem. Eng. Asp. 2016, 506, $298-305$. [CrossRef]

4. Cuce, E.; Cuce, P.M.; Wood, C.J.; Riffat, S.B. Toward aerogel based thermal superinsulation in buildings: A comprehensive review. Renew. Sustain. Energy Rev. 2014, 34, 273-299. [CrossRef]

5. Zu, G.Q.; Shen, J.; Wang, W.Q.; Zou, L.P.; Lian, Y.; Zhang, Z.H. Silica-titania composite aerogel photocatalysts by chemical liquid deposition of titania onto nanoporous silica scaffolds. ACS Appl. Mater. Interfaces 2015, 7, 5400-5409. [CrossRef] [PubMed]

6. Alnaief, M.; Smirnova, I. Effect of surface functionalization of silica aerogel on their adsorptive and release properties. J. Non-Cryst. Solids 2010, 356, 1644-1649. [CrossRef]

7. Ciriminna, R.; Fidalgo, A.; Pandarus, V.; Beland, F.; Ilharco, L.M.; Pagliaro, M. The sol-gel route to advanced silica-based materials and recent applications. Chem. Rev. 2013, 113, 6592-6620. [CrossRef] [PubMed]

8. Wong, J.C.H.; Kaymak, H.; Brunner, S.; Koebel, M.M. Mechanical properties of monolithic silica aerogels made from polyethoxydisiloxanes. Microporous Mesoporous Mater. 2014, 183, 23-29. [CrossRef]

9. Liu, G.W.; Zhou, B.; Du, A.; Shen, J.; Wu, G.M. Greatly strengthened silica aerogels via co-gelation of binary sols with different concentrations: A method to control the microstructure of the colloids. Colloids Surf. A Physicochem. Eng. Asp. 2013, 436, 763-774. [CrossRef]

10. Wang, Z.; Dai, Z.; Wu, J.J.; Zhao, N.; Xu, J. Vacuum-dried robust bridged silsesquioxane aerogels. Adv. Mater. 2013, 25, 4494-4497. [CrossRef] [PubMed]

11. Yun, S.; Luo, H.J.; Gao, Y.F. Low-density, hydrophobic, highly flexible ambient-pressure-dried monolithic bridged silsesquioxane aerogels. J. Mater. Chem. A 2015, 3, 3390-3398. [CrossRef]

12. Rao, A.V.; Kulkarni, M.M.; Amalnerkar, D.P.; Seth, T. Superhydrophobic silica aerogels based on methyltrimethoxysilane precursor. J. Non-Cryst. Solids 2003, 330, 187-195.

13. Cai, L.; Shan, G.R. Elastic silica aerogel using methyltrimethoxysilane precusor via ambient pressure drying. J. Porous Mater. 2015, 22, 1455-1463. [CrossRef]

14. Kanamori, K.; Aizawa, M.; Nakanishi, K.; Hanada, T. New transparent methylsilsesquioxane aerogels and xerogels with improved mechanical properties. Adv. Mater. 2007, 19, 1589-1593. [CrossRef]

15. Kanamori, K. Monolithic silsesquioxane materials with well-defined pore structure. J. Mater. Res. 2014, 20, 2773-2786. [CrossRef]

16. Hayase, G.; Kugimiya, K.; Ogawa, M.; Kodera, Y.; Kanamori, K.; Nakanishi, K. The thermal conductivity of polymethylsilsesquioxane aerogels and xerogels with varied pore sizes for practical application as thermal superinsulators. J. Mater. Chem. A 2014, 2, 6525-6531. [CrossRef]

17. Lei, C.S.; Hu, Z.J.; Zhang, Y.; Yang, H.L.; Li, J.N.; Hu, S.B. Tailoring structural and physical properties of polymethylsilsesquioxane aerogels by adjusting $\mathrm{NH}_{3} \cdot \mathrm{H}_{2} \mathrm{O}$ concentration. Microporous Mesoporous Mater. 2018, 258, 236-243. [CrossRef]

18. Ingale, S.V.; Wagh, P.B.; Tripathi, A.K.; Kamble, V.S.; Kumar, R.; Gupta, S.C. Physico-chemical properties of silica aerogels prepared from TMOS/MTMS mixtures. J. Porous Mater. 2011, 18, 567-572. [CrossRef]

19. Rao, A.V.; Pajonk, G.M. Effect of methyltrimethoxysilane as a co-precursor on the optical properties of silica aerogels. J. Non-Cryst. Solids 2001, 285, 202-209. 
20. Cheng, X.L.; Chen, D.R.; Liu, Y.J. Mechanisms of silicon alkoxide hydrolysis-oligomerization reactions: A DFT investigation. Chem. Phys. Chem. 2012, 13, 2392-2404. [CrossRef] [PubMed]

21. Tamon, H.; Sone, T.; Okazaki, M. Control of mesoporous structure of silica aerogel prepared from TMOS. J. Colloid Interface Sci. 1997, 188, 162-167. [CrossRef]

22. Wu, G.Y.; Yu, Y.X.; Cheng, X.; Zhang, Y. Preparation and surface modification mechanism of silica aerogels via ambient. Mater. Chem. Phys. 2011, 129, 308-314. [CrossRef]

23. Sanli, D.; Erkey, C. Monolithic composites of silica aerogels by reactive supercritical deposition of hydroxy-terminated poly(dimethylsiloxane). ACS Appl. Mater. Interfaces 2013, 5, 11708-11717. [CrossRef] [PubMed]

24. Zu, G.Q.; Shen, J.; Zou, L.P.; Wang, W.Q.; Lian, Y.; Zhang, Z.H.; Du, A. Nanoengineering super heat-resistant, strong alumina aerogels. Chem. Mater. 2013, 25, 4757-4764. [CrossRef]

25. Bhagat, S.D.; Kim, Y.-H.; Ahn, Y.-S.; Yeo, J.-G. Rapid synthesis of water-glass based aerogels by in situ surface modification of the hydrogels. Appl. Surf. Sci. 2007, 253, 3231-3236. [CrossRef]

26. Wang, W.Q.; Zhang, Z.H.; Zu, G.Q.; Shen, J.; Zou, L.P.; Lian, Y.; Liu, B.; Zhang, F. Trimethylethoxysilanemodified super heat-resistant alumina aerogels for high-temperature thermal insulation and adsorption applications. RSC Adv. 2014, 4, 54864-54871. [CrossRef]

27. Zu, G.Q.; Shen, J.; Zou, L.P.; Zou, W.B.; Guan, D.Y.; Wu, Y.; Zhang, Y.W. Highly thermally stable zirconia/silica composite aerogels prepared by supercritical deposition. Microporous Mesoporous Mater. 2017, 238, 90-96. [CrossRef]

28. Li, Z.; Cheng, X.D.; He, S.; Huang, D.M.; Bi, H.J.; Yang, H. Preparation of ambient pressure dried MTMS/TEOS co-precursor silica aerogel by adjusting $\mathrm{NH}_{4} \mathrm{OH}$ concentration. Mater. Lett. 2014, 129, 12-15. [CrossRef]

29. Chang, K.-J.; Wang, Y.-Z.; Peng, K.-C.; Tsai, H.-S.; Chen, J.-R.; Huang, C.-T.; Ho, K.-S.; Lien, W.-F. Preparation of silica aerogel/polyurethane composites for the application of thermal insulation. J. Polym. Res. 2014, 21, 1-9. [CrossRef]

30. Mazraeh-shahi, Z.T.; Shoushtari, A.M.; Abdouss, M.; Bahramian, A.R. Relationship analysis of processing parameters with micro and macro structure of silica aerogel dried at ambient pressure. J. Non-Cryst. Solids 2013, 376, 30-37. [CrossRef]

31. Lin, Y.-F.; Hsu, S.-H. Solvent-resistant CTAB-modified polymethylsilsesquioxane aerogels for organic solvent and oil adsorption. J. Colloid Interface Sci. 2017, 485, 152-158. [CrossRef] [PubMed]

32. Li, T.M.; Zhou, B.; Du, A.; Xiang, Y.L.; Wu, S.; Liu, M.F.; Ding, W.H.; Shen, J.; Zhang, Z.H. Microstructure control of the silica aerogels via pinhole drying. J. Sol-Gel Sci. Technol. 2017, 84, 96-103. [CrossRef]

33. Yokogawa, H. Transparent silica aerogel blocks for high-energy physics research. In Aerogels Handbook, 28th ed.; Aegerter, M.A., Levetis, N., Koebel, M.M., Eds.; Springer: New York, NY, USA, 2011; pp. 651-664.

34. Zhao, S.Y.; Zhang, Z.; Sèbe, G.; Wu, R.; Virtudazo, R.V.R.; Tingaut, P.; Koebel, M.M. Multiscale assembly of superinsulating silica aerogels within silylated nanocellulosic scaffolds: Improved mechanical properties promoted by nanoscale chemical compatibilization. Adv. Funct. Mater. 2015, 25, 2326-2334. [CrossRef]

35. Sai, H.Z.; Fu, R.; Xing, L.; Xiang, J.H.; Li, Z.Y.; Li, F.; Zhang, T. Surface modification of bacterial cellulose aerogels' web-like skeleton for oil/water separation. ACS Appl. Mater. Interfaces 2015, 7, 7373-7381. [CrossRef] [PubMed]

Sample Availability: Samples of the PMSQ aerogels and the modified samples are available from the authors. 